\title{
Atherosclerosis: pathogenesis and increased occurrence in individuals with HIV and Mycobacterium tuberculosis infection
}

This article was published in the following Dove Press journal:

HIVIAIDS - Research and Palliative Care

28 October 2010

Number of times this article has been viewed

\author{
Timothy Guilford' \\ Devin Morris ${ }^{2,4}$ \\ Dennis Gray ${ }^{3,4}$ \\ Vishwanath Venketaraman ${ }^{3,4}$ \\ 'Your Energy Systems, Palo Alto, \\ CA, USA; ${ }^{2}$ Graduate of College of \\ Biomedical Sciences, ${ }^{3}$ College of \\ Osteopathic Medicine of the Pacific, \\ ${ }^{4}$ Western University of Health \\ Sciences, Pomona, CA, USA
}

Correspondence: Vishwanath Venketaraman

Department of Basic Medical Sciences, College of Osteopathic Medicine of the Pacific, Western University of Health Sciences, 309 East Second Street,

Pomona, CA 91766, USA

Tel +l 909-706-3736

Email vvenketaraman@westernu.edu

\begin{abstract}
Atherosclerosis is a leading cause of coronary heart disease and stroke. Since 1981, more than 980,000 cases of AIDS have been reported in the United States. According to the Centers for Disease Control, more than 1 million Americans may be infected with HIV. By killing or damaging CD4+ T cells of the body's immune system, HIV progressively destroys the body's ability to fight infections. People diagnosed with AIDS often suffer from life-threatening diseases caused by opportunistic infections such as tuberculosis. HIV-infected individuals have increased risks for atherosclerosis. This review summarizes the effects of oxidized low density lipoproteins in impairing macrophage functions in individuals with atherosclerosis (with and without HIV infection) thereby enhancing the susceptibility to Mycobacterium tuberculosis infection.
\end{abstract}

Keywords: AIDS, HIV, Mycobacterium tuberculosis

\section{Introduction}

Oxidized low-density lipoprotein (ox-LDL) has been shown in several studies to be an independent marker of the progression of atherosclerosis. ${ }^{1-4}$ The pathophysiology relates to macrophage ingestion of excess ox-LDL and the formation of foam cells, the acknowledged trigger of atherosclerosis. ${ }^{5}$ It has been shown that both highdensity lipoprotein (HDL) and low-density lipoprotein (LDL) cholesterol contain the antioxidant enzyme glutathione peroxidase embedded in the lipoprotein, and a continuous supply of glutathione (GSH) is needed to prevent the oxidation of HDL and LDL cholesterol. ${ }^{6}$ The observation that excessive ox-LDL ingestion by macrophage interferes with normal macrophage function may have implications in other disease conditions in which low GSH plays a role, such as HIV infection. Individuals with HIV infection are at increased risk for the progression of atherosclerosis ${ }^{7}$ as well as infectious diseases such as tuberculosis (TB). ${ }^{8}$ This review postulates that the compromise in macrophage function that occurs with HIV may increase the risk of both atherosclerosis as well as susceptibility to Mycobacterium tuberculosis $(M . t b)$ infection. The increase in ox-LDL ingestion and resulting macrophage dysfunction may contribute to the increased risk for $M$. $t b$ infection in both HIV-positive and HIV-negative individuals.

\section{Atherosclerosis}

Atherosclerosis is a leading cause of coronary heart disease and stroke, which were responsible for more than 589,000 deaths in 2005 - almost $25 \%$ of all deaths in the United States. Each year more than 1.2 million will suffer a coronary attack and almost 
800,000 people will suffer a stroke. Atherosclerosis is a process in which deposits of fatty substances, cholesterol, cellular waste products, calcium and other substances build up in the inner lining of an artery. This build-up is called plaque. $^{7}$ It usually affects large- and medium-sized arteries. Some hardening of arteries often occurs when people grow older. Plaques can grow large enough to significantly reduce the blood's flow through an artery. But most of the damage occurs when they become fragile and rupture. Plaques that rupture cause blood clots to form that can block blood flow or break off and travel to another part of the body. If either happens and blocks a blood vessel that feeds the heart, it causes a heart attack. And if blood supply to the arms or legs is reduced, it can cause difficulty walking and eventually lead to gangrene. Atherosclerosis can result in myocardial infarction, and bits of plaque can lodge in arteries in the brain, causing a stroke. ${ }^{7}$

\section{Glutathione}

GSH is a tripeptide that, in its reduced form, protects cells against oxidizing agents, free radicals and reactive oxygen intermediates (ROI). In addition to its antioxidant role, GSH plays a vital role in maintenance of cell viability and regulating immune cell functions. ${ }^{9}$ Synthesis of GSH occurs in 2 steps. The initial step (the rate limiting step) is the formation of a dipeptide, $\gamma$-glutamyl cysteine, a reaction that is catalyzed by $\gamma$-glutamylcysteine synthetase. Intracellular levels of L-cysteine are substantially lower than levels of L-glutamate and glycine. Therefore, GSH synthesis is limited by the availability of cysteine. ${ }^{10}$ The second step involved in the synthesis of GSH is the formation of $\gamma$-glutamyl cysteine glycine, catalyzed by the enzyme GSH synthetase. ${ }^{10}$ Intracellular GSH levels can be increased or decreased by treatment with $\mathrm{N}$-acetyl cysteine (NAC) or buthionine sulfoximine (BSO), respectively. The most efficient way to increase the levels of cysteine in cells grown in vitro is to supply the culture medium with NAC, which is easily taken up by the cells and is nontoxic. Intracellularly, NAC is de-acetylated and cysteine is utilized for GSH synthesis. BSO specifically inhibits the activity of the $\gamma$-glutamyl-cysteinyl synthetase enzyme, which catalyzes the first step reaction in the synthesis of GSH,,${ }^{9,10}$ leading to inhibition in the synthesis of GSH.

\section{Oxidized LDL}

Oxidation of LDL (ox-LDL) cholesterol has been shown to convert LDL cholesterol to a form that is recognized by scavenger receptors on macrophages and to contribute to foam cell formation. ${ }^{6}$ It has been known for some time that vitamin $E$ and $\beta$-carotene are found in the LDL complex and that decreases in vitamin $\mathrm{E}$ and $\beta$-carotene are early events reflecting the initial stages of lipid peroxidation formation. ${ }^{11}$ It has only recently been shown that glutathione peroxidase (GPx) is also found to occur naturally in the LDL lipoprotein. Substituting the unique substrate for GPx, reduced GSH, with liposomal GSH can slow the formation of ox-LDL in vitro in human blood and slow atherosclerosis in vivo in the $\mathrm{ApoE}^{(-/)}$mouse model of atherosclerosis. $^{12}$

\section{Ox-LDL and atherosclerosis}

Ox-LDL has been shown in several studies to be an independent marker of the progression of atherosclerosis. ${ }^{1-4}$ The pathophysiology relates to macrophage ingestion of excess ox-LDL and the formation of foam cells, the acknowledged trigger of atherosclerosis. ${ }^{5}$ The mechanism of ox-LDL and the requirement for GSH to prevent oxidation of LDL cholesterol has been described in Rosenblat et al ${ }^{12}$ using liposomal GSH as the source of GSH in both human blood, in vitro studies and in the mouse model of atherosclerosis. This study demonstrated that both LDL and HDL contain the antioxidant enzyme GSH peroxidase embedded in the lipoprotein. ${ }^{12}$ As GSH is the single substrate for GSH peroxidase, a continuous supply of GSH in the reduced state is needed to prevent the oxidation of LDL and HDL cholesterol. This study also demonstrated that preventing the oxidation of LDL and increasing the intracellular GSH levels is able to maintain normal macrophage function. It is likely that the presence of excess amounts of ox-LDL will be an indicator of general macrophage dysfunctions as it has been shown to stimulate the release of macrophage inflammatory protein 1-alpha (MIP-1 alpha) ${ }^{5}$ as well as other immune responses. Ox-LDL is known to be ingested by macrophages and without adequate antioxidant function is prone to form foam cells (Figure 1), a transition which is also a contributing factor to the formation of atherosclerosis ${ }^{14}$ and atherosclerotic lesions. ${ }^{13-15}$

\section{Macrophages and atherosclerosis}

Macrophages are derived from the circulating pool of monocytes. ${ }^{16}$ Monocytes are produced in the bone marrow and, in the absence of specific survival signals, are programmed to undergo apoptosis in 24-48 hours. ${ }^{16,17}$ When monocytes are recruited into local environments they differentiate into macrophages. In arteries, macrophage scavenging function may be diverted to the formation of plaque; in the lung 


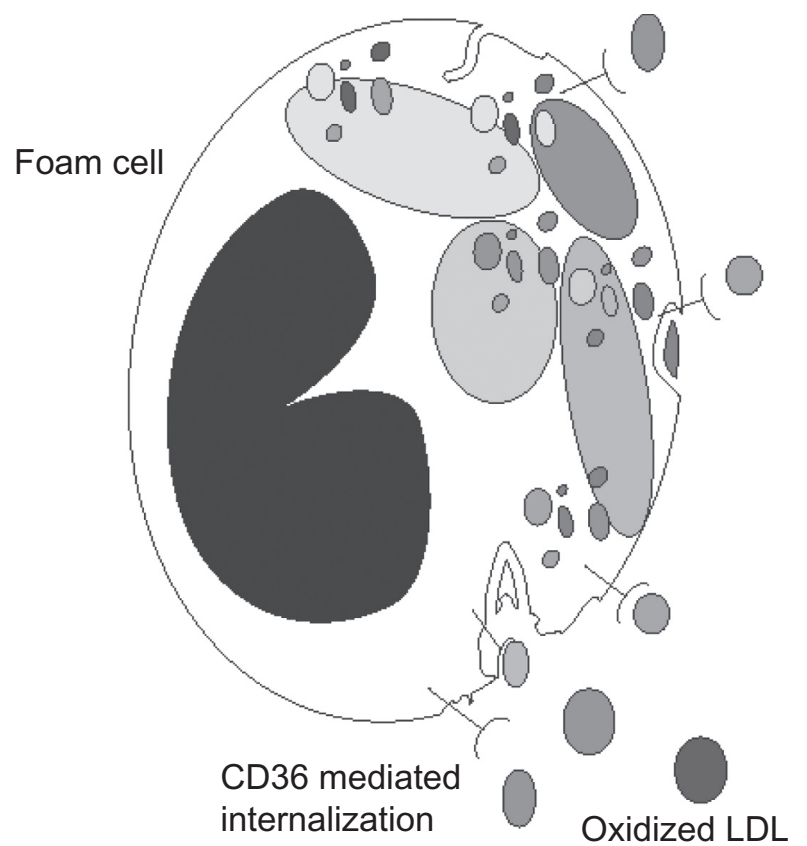

Figure I Receptor mediated uptake of oxidized low density lipoproteins by macrophages resulting in foam cell formation.

they become alveolar macrophages. In atherosclerosis, macrophage oxidative impairment occurs in the GSH-related antioxidant system, ${ }^{5}$ probably related to the unregulated ingestion of ox-LDL via the CD36 receptor. ${ }^{18,19}$

\section{Role of GSH in macrophage function}

In the natural state, GSH levels are maintained by both the synthesis of GSH and by GSH reductase, an enzyme that catalyzes the reduction of glutathione disulfide (GSSG) to GSH, with NADPH as the reducing cofactor. Increasing the expression of GSH reductase, the enzyme which returns GSH to the biochemically reduced (functional) state, decreases atherosclerotic lesions. ${ }^{20}$ It has been shown that lymphocyte proliferation after mitogenic lectin exposure is decreased ${ }^{21}$ with low intracellular GSH. ${ }^{21,22}$

The level of reduced GSH in the extracellular lung fluid (ELF) or alveolar lining fluid has been estimated to be as much as 140-fold higher than the level of reduced GSH found in the plasma of the same individuals. ${ }^{23}$ The antioxidant GSH is essential for the detoxification of endogenous and exogenous oxidant radicals and protection of cells residing in the airway and alveolus ${ }^{24,25}$ and during oxidative stress such as cigarette smoking. ${ }^{26}$ In disease states, the level of ELF GSH may decrease dramatically to the point that acute respiratory distress syndrome has only $0.05 \%$ of the amount found in the normal ELF. ${ }^{27}$
In the lung, intracellular antioxidants are expressed at a relatively low level in the lung epithelial tissue and are not induced by oxidative stress. The major antioxidants in the lung are extracellular and the GSH system is the major antioxidant mechanism used in the airways. ${ }^{28}$ Alveolar macrophage cells rely on the ELF level of GSH to provide the biochemical support to maintain intracellular GSH levels ${ }^{29}$ and protect their membranes during the respiratory burst. ${ }^{30}$

When macrophage GSH availability is limited, cellular functions such as phagocytosis and microbial clearance are compromised. ${ }^{24}$ The mouse model of decreased alveolar macrophage GSH resulted in a decrease in the rate of phagocytosis of individual macrophages as well as a decrease in the overall percentage of macrophages positive for phagocytosis. ${ }^{24}$ Similar findings have been reported in the alveolar function of guinea pigs exposed to ethanol in utero resulting in low macrophage GSH. The addition of precursors of GSH to the diet of the ethanol-exposed animals increased the GSH levels in the ELF and alveolar macrophages and maintained both the rate of internalization and the number of macrophages positive for internalization. ${ }^{24}$ It was also shown that decreased GSH availability in the ELF and alveolar macrophages of ethanol-fed mice resulted in an increase of apoptosis of macrophages that could be reversed by maintaining GSH availability. ${ }^{24}$

\section{GSH and arterial macrophage functions in atherosclerosis}

GSH deficiency in the macrophage has been shown to be associated with compromise of mitochondrial function. GSH plays an integral role in protecting the mitochondria from ROI damage and it is the level of mitochondrial GSH that determines when cellular toxicity commences. ${ }^{18}$ GSH deficiency in mitochondria contributes to macrophage dysfunction in atherosclerosis. Data from a study of the upregulation of GSH reductase in macrophages provide direct evidence that the GSH-dependent anti-oxidant system in macrophages plays a critical role in atherogenesis. ${ }^{20}$ The study shows that overexpression of GSH in either mitochondria or the cytosol of macrophages decreases the severity of atherosclerosis in this animal model. ${ }^{20}$

Ox-LDL-related peroxyl radical formation is implicated in both mitochondrial dysfunction and macrophage lysis. ${ }^{19}$ Ox-LDL toxicity is related to compromise of mitochondrial function including an increase in mitochondrial $\mathrm{Ca}^{+2}$, opening of mitochondrial permeability transition pores and depolarization of mitochondrial membrane potential. 
It has been observed that maintaining adequate GSH levels in macrophage will prevent the toxicity of ox-LDL in an in vitro macrophage model. ${ }^{31-33}$ These findings help explain why a decreased amount of reduced GSH in blood has been shown to be an independent marker of progression of atherosclerosis in 2 studies. ${ }^{13,14}$

\section{HIV}

Worldwide, it is estimated that approximately 40 million people are infected with HIV, two-thirds of whom live in sub-Saharan Africa. Three regions, Africa, Asia, and Latin America, have the highest rates of new infections. AIDS is the fourth leading cause of death worldwide. ${ }^{34} \mathrm{HIV}$ is the cause of AIDS. Both HIV-1 and HIV-2 cause AIDS, but HIV-1 is found worldwide, whereas HIV-2 is found primarily in West Africa. HIV preferentially infects and kills CD4+ T lymphocytes, resulting in the loss of cell-mediated immunity and a high probability that the host will develop opportunistic infections. HIV-infected individuals have an increased risk for atherosclerosis and susceptibility to $M . t b$ infection. ${ }^{35}$

\section{HIV and atherosclerosis}

As people with HIV live longer due to effective combination antiretroviral therapy (ART), cardiovascular disease has become an increasingly important cause of morbidity and mortality. But it remains controversial whether HIV infection contributes to accelerated atherosclerosis independent of traditional cardiovascular risk factors. In a recent crosssectional study, more than $400 \mathrm{HIV}$-positive participants without pre-existing cardiovascular disease in the FRAM (Fat Redistribution and Metabolic Change in HIV Infection) study were compared with HIV-negative participants in the MESA (Multi-Ethnic Study of Atherosclerosis) cohort. ${ }^{7}$ The preclinical atherosclerosis was assessed by measuring carotid intima-media thickness (IMT), or thickness of the walls of the arteries in the neck that supply the brain. IMT was evaluated at 2 sites in the artery, known as the internal/ bulb. Even after adjustment for traditional cardiovascular disease risk factors, HIV infection was accompanied by more extensive atherosclerosis as measured by IMT. ${ }^{7}$ The association of HIV infection with IMT was similar to that of traditional cardiovascular disease risk factors, such as smoking. The impact of HIV infection on preclinical atherosclerosis overrides any small differences related to antiretroviral therapy or specific drug classes. The effect of HIV is so big that no drug or class of drugs stands out as being an effective contributor. ${ }^{7}$

\section{GSH and HIV-infection}

Findings from our research laboratory as well as by other groups confirm that intracellular levels of GSH are decreased in patients with AIDS, ${ }^{35}$ in whom the risk of TB is many times that of healthy individuals. ${ }^{35}$ The factors responsible for low GSH in HIV infection are poorly understood. Infection with $\mathrm{HIV}$ is believed to trigger a range of metabolic changes in addition to the progressive deficits in cellular immunity and increased susceptibility to opportunistic infections that are its clinical hallmarks, and the progression to AIDS. ${ }^{36-45}$ The decreased GSH content in immune cells of HIV-positive individuals was at least in part attributed to the decrease in plasma cysteine and increased plasma glutamate (an inhibitor of cysteine permeation via the Xc-transport system), as observed during early infection (Figure 2). The decreased intracellular GSH and plasma cysteine observed in HIV patients may also be due to chronic oxidative stress and this may lead to the progression of the disease. The decreased availability of cysteine can be overcome to some extent by the cysteine precursor NAC. ${ }^{42}$ Herzenberg and colleagues found that NAC treatment improves the clinical situation and delays

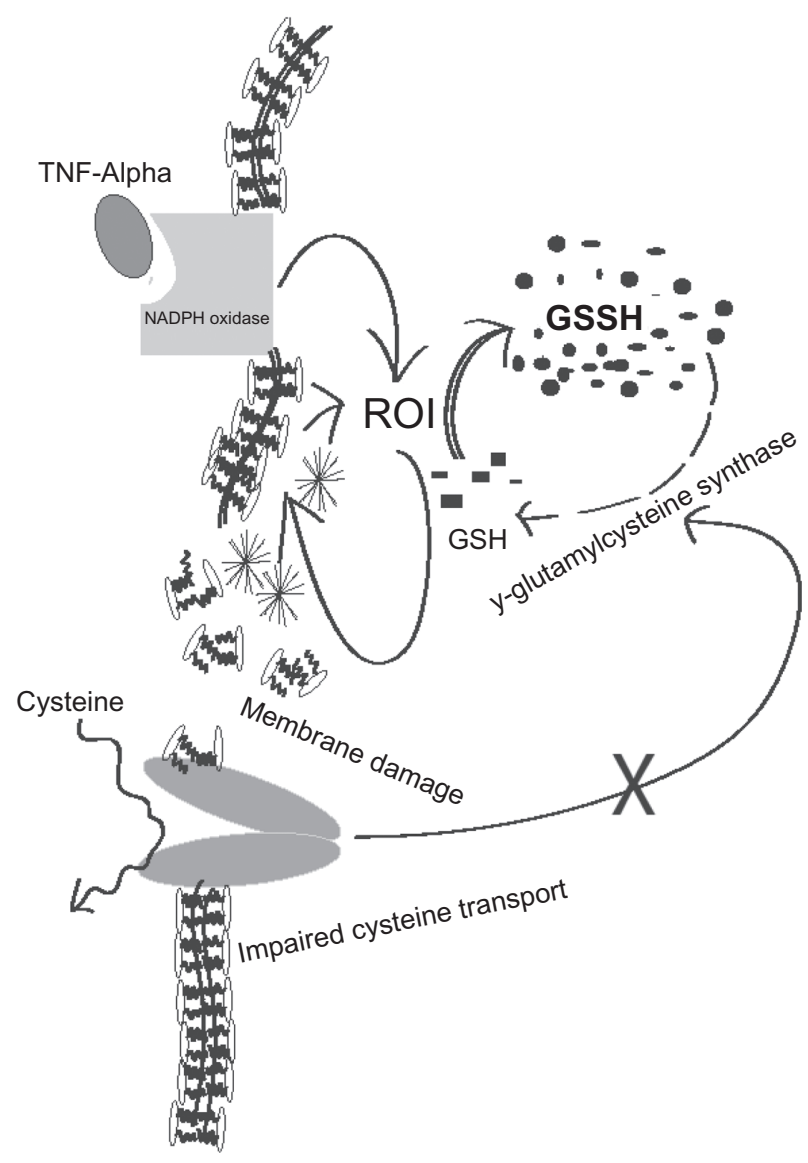

Figure 2 Possible causes for decreased intracellular levels of glutathione in individuals with HIV and/or M. tb infection. 
the disease progression. ${ }^{43}$ This study showed that long-term NAC administration to AIDS patients improves their hematological profile, GSH content and life expectancy. ${ }^{43}$

\section{Ox-LDL and HIV}

Increased incidence of cardiovascular events in HIV patients has sparked interest in elucidating the molecular mechanism behind endothelial dysfunction resulting in atherosclerotic pathology. While there is no evidence within the literature of a molecular mechanism responsible for increased cardiovascular risk within such HIV+ cohorts, animal models expressing transgenic HIV-1 offer a promising insight. One such study has shown significantly increased aortic arch lectin-like oxidized-low-density-lipoprotein receptor-1 (LOX-1) gene expression in HIV-1 transgenic rats compared to control rats. ${ }^{46} \mathrm{LOX}-1$ is an endothelial receptor for ox-LDL and is hypothesized to be an early marker of endothelial dysfunction. It has been proposed that endothelial dysfunction is the first step in progression towards atherosclerotic plaque formation. This same group has also demonstrated significantly increased expression of inflammatory endothelial adhesion molecules in the same HIV-1 model. Both inducible vascular cell adhesion molecule-1 (VCAM-1) and constitutively expressed intercellular adhesion molecule-1 (ICAM-1) were both significantly elevated in HIV-1 transgenic rats compared to control rats, strongly suggesting for the first time that the HIV infection, independent of antiretroviral therapy-associated dyslipidemia, is capable of pathologic endothelial dysfunction that may prelude atherosclerotic plaque formation in major blood vessels. $^{46}$

\section{Tuberculosis}

TB is the most prevalent infectious disease in the world. ${ }^{47-54}$ In recent years there has been a significant increase in the incidence of TB due to the emergence of multi-drug resistant strains of $M$. $t b$ and the increased numbers of highly susceptible immuno-compromised individuals arising from the AIDS pandemic. ${ }^{8}$ It is also believed that in developing countries, as many as $40 \%-80 \%$ of individuals with AIDS are at risk of developing TB. ${ }^{8,35}$ The innate and adaptive immune systems contribute to host defenses against $M$. $t b$ infection. ${ }^{48-54}$ Control of $M$. $t b$ infection occurs both at the macroscopic and cellular levels. At the macroscopic level, the physical containment of viable mycobacteria within fibrotic granulomas contributes to prevention of overt disease. ${ }^{49-50}$ Control of mycobacterial replication also occurs within macrophages. Resident tissue macrophages provide the first-line defense against $M . t b$ infection. Macrophages and natural killer (NK) cells play an important role in innate defense against $M . t b$ infection. ${ }^{51-53}$

\section{Tuberculosis and GSH}

Our group reported that GSH plays a key role in limiting intracellular growth of Mycobacterium bovis BCG in nitric oxide (NO)-deficient macrophages, such as macrophages derived from inducible nitric oxide synthase (NOS2)-knock out mice and human peripheral blood monocyte-derived macrophages (HMDM). Thus, GSH has direct antimycobacterial activity distinct from its role as an NO carrier, functioning as an effector molecule in innate defense against $M$. $t b$ infection. ${ }^{47,51,52}$ These results unfold a novel and potentially important innate defense mechanism adopted by human macrophages to control $M$. $t b$ infection. ${ }^{51,52}$ Consistent with these observations, we have also found that GSH mediates growth control of virulent $M$. $t b$ in human blood cultures. ${ }^{35,53}$ These results indicate that the inability of immune cells to contain $M . t b$ growth may be a consequence of the inability of the immune cells to maintain adequate GSH levels during in vitro infection. ${ }^{35}$ Furthermore, our recent studies indicate that GSH in combination with cytokines such as interleukin (IL-2) and IL-12, activate NK cells to control $M$. $t b$ infection. ${ }^{53}$ Most importantly, our recent studies demonstrate that GSH levels are significantly reduced in peripheral blood mononuclear cells and red blood cells isolated from TB patients and this decrease correlates with increased proinflammatory cytokine production and enhanced growth of M. $t b .{ }^{54}$ Our group was the first to report that GSH levels are decreased in individuals with pulmonary $\mathrm{TB}$ and to correlate GSH levels with protective immunity. ${ }^{54}$ Furthermore, our study provides a direct relationship between decreased GSH levels, pro-inflammatory cytokine production and enhanced growth of $M . t b$ in TB patients. ${ }^{54}$

\section{ROI, GSH and TB}

ROI are generated from NADPH through a catalytic reaction of membrane bound enzyme NADPH oxidase, which is activated by assembling cytosolic regulatory components. The most important characteristic of ROI, either in vivo or in vitro, is peroxidation of lipids resulting in tissue damage and death of affected cells. Lipid peroxidation is a chain reaction providing a continuous supply of free radicals that initiate further peroxidation. ${ }^{55}$ Free radical-induced lipid peroxidation causes marked alterations in the structural integrity and functions of membrane. The lipid peroxides formed at the primary site such as lungs could be transferred through 
the circulation to other organs and tissues. ${ }^{56-58}$ Jack et al ${ }^{59}$ reported that several circulating markers of free radical activity were increased in pulmonary TB patients and some of these markers remain elevated even after completion of antimicrobial chemotherapy, indicating ongoing oxidative stress, which may contribute to decreased GSH levels. It is our prediction that in active TB, increased levels of ROI cause impaired cystine transport leading to decreased levels of GSH (Figure 2).

\section{TNF- $\alpha$, ROI and GSH}

It has been shown that TNF- $\alpha$ stimulates ROI production. TNF- $\alpha$ could impair GSH-redox status by production of ROI, and impairment of the GSH-reductase system, thereby leading to decreased regeneration of reduced GSH from oxidized GSSG (Figure 2). Moreover, enhanced ROI is likely to increase TNF- $\alpha$ in various cells, and depletion of reduced GSH will increase the inflammatory response to the cytokine. Nuclear factor-kappa B (NF- $\kappa \mathrm{B})$ is a DNA binding protein and a ROI-sensitive transcription factor for several cytokines, including TNF- $\alpha .{ }^{57}$ While NF-kB activation (leading to TNF- $\alpha$ production) is induced and enhanced by ROI, it can also be blocked by anti-oxidants such as vitamin E and GSH-enhancing agents such as NAC. ${ }^{57}$ Excessive production of TNF- $\alpha$ and increased tissue sensitivity to this cytokine has been implicated in the immunopathology ofTB, such as caseous necrotizing reactions. For example, excess TNF- $\alpha$ (relative to its receptors) in human bronchoalveolar lavage fluid was associated with tissue necrosis and cavity formation. ${ }^{59-61}$ Systemic spillover of TNF- $\alpha$ may account for unwanted inflammatory effects like fever and wasting, which manifests clinically as cachexia, consistent with the original designation as cachetin. ${ }^{65}$ In HIV-infected persons, the effects of TNF- $\alpha$ may be particularly deleterious, as this cytokine has been implicated as a stimulus for HIV expression and for activation-induced $\mathrm{T}$ cell apoptosis. For these reasons, several studies have examined anti-TNF treatments in persons with AIDS and in HIV+ TB. ${ }^{62-65}$ Thus enhanced TNF- $\alpha$ production may represent a pathogenic loop, leading to enhanced inflammation and ROI production, leading to reduced GSH levels.

\section{Atherosclerosis and tuberculosis}

Atherosclerosis is an inflammatory disease involving the accumulation of macrophages in the intima. Wnt5a is a noncanonical member of the Wnt family of secreted glycoproteins. Recently, human macrophages have been shown to express Wnt5a upon stimulation with bacterial pathogens in vitro and in granulomatous lesions in the lung of $M$. $t b$-infected patients. Wnt5a expression has also been linked to Toll-like receptor-4 (TLR-4), an innate immune receptor implicated in atherosclerosis. These observations, along with the fact that Wnt5a is involved in cell migration and proliferation, led Christman et al to postulate that Wnt5a plays a role in atherosclerosis. ${ }^{66}$ To investigate this hypothesis, Christman et al characterized Wnt5a expression in murine and human atherosclerotic lesions. Tissue sections derived from the aortic sinus to the aortic arch of apolipoprotein E-deficient mice and sections derived from the carotid arteries of patients undergoing endarterectomy were subjected to immunohistochemical analysis. All samples were found to be positive for Wnt5a with predominant staining in the areas of macrophage accumulation within the intima. In parallel, the investigators probed for the presence of TLR-4 and found coincident TLR-4 and Wnt5a expression. ${ }^{66}$ For both the Wnt5a and TLR-4 staining, consecutive tissue sections treated with an isotype- and species-matched Ig served as a negative control and exhibited little, if any, reactivity. Quantitative RT-PCR revealed that Wnt5a mRNA expression in RAW264.7 murine macrophages can be induced by stimulation with LPS, a known ligand for TLR-4. Combined, these findings demonstrate for the first time Wnt5a expression in human and murine atherosclerotic lesions and suggest that cross-talk between TLR-4 and Wnt5a is operative in atherosclerosis. ${ }^{66}$

\section{Conclusion}

Macrophage functions are compromised by decreased intracellular levels of GSH when an excess of ox-LDL is present. This has been shown to increase the development and progression of atherosclerosis. Reduction of intracellular GSH is a feature of HIV, which is also associated with an increased risk of atherosclerosis. The compromise of innate immune function that occurs with low GSH in the macrophage may also increase susceptibility to infection with $M$. $t b$, for which individuals with HIV have an increased risk. It is possible that the compromise of macrophage function related to the ingestion of ox-LDL and the decreased macrophage levels of GSH increase the risk of contracting macrophage-related infectious disease such as $M$. $t b$ infection in both HIV-positive and HIV-negative individuals. These observations suggest that further investigation into the relationship between low GSH, increased ox-LDL and susceptibility to infection with $M$. $t b$ is warranted.

\section{Acknowledgment}

Corresponding author's work is supported by American Heart Association (Scientist Development Grant), Potts 
Memorial Foundation, UMDNJ Foundation grant, Your Energy Systems and start-up funds from Western University of Health Sciences.

\section{Disclosure}

The authors declare no conflicts of interest.

\section{References}

1. Gomez M, Valle V, Aros F, Sanz G, Sala J, Fiol M, et al. Oxidized LDL, lipoprotein (a) and other emergent risk factors in acute myocardial infarction (FORTIAM study). Rev Esp Cardiol. 2009;62(4): 373-382.

2. Naruko T, Ueda M, Ehara S, Itoh A, Haze K, Shirai N, et al. Persistent high levels of plasma oxidized low-densty lipoprotein after acute myocardial infarction predict stent restenosis. Arterioscler Thromb Vasc Biol. 2006;26(4):877-883.

3. Meisinger C, Baumert J, Khuseyinova N, Loewel H, Koenig W. Plasma oxidized low-density lipoprotein, a strong predictor for acute coronary heart disease events in apparently healthy, middle-aged men from the general population. Circulation. 2005;112(5):651-657.

4. Yamashita H, Ehara S, Yoshiyama M, Naruko T, Haze K, Shirai N, et al. Elevated plasma levels of oxidized low-density lipoprotein relate to the presence of angiographically detected complex and thrombotic coronary artery lesion morphology in patients with unstable angina. Circ J. 2007;71(5):681-687.

5. Pentikainen MO, Oorni K, Ala-Korpela M, Kovanen PT. Modified LDL - trigger of atherosclerosis and inflammation in the arterial intima. J Internal Med. 2000;247(3):359-370.

6. Steinbrecher UP, Witztum JL, Parthasarathy S, Steinberg D. Decrease in reactive amino groups during oxidation or endothelial cell modification of LDL. Correlation with changes in receptor-mediated catabolism. Arterioscler Thromb Vasc Biol. 1987;7(2):135-143.

7. Grunfeld C, Delaney JA, Wanke C, et al. Preclinical atherosclerosis due to HIV infection: carotid intima-medial thickness measurements from the FRAM study. AIDS. 2009;23(14):1841-1849.

8. Havlir DV, Getahun H, Sanne I, Nunn P. Opportunities and challenges for HIV care in overlapping HIV and TB epidemics. JAMA. 2008;300(4): 423-430.

9. Deneke SM, Fanburg BL. Regulation of cellular glutathione. Am J Physiol. 1989;257(4 Pt 1):L163-L173.

10. Griffith OW. Biologic and pharmacologic regulation of mammalian glutathione synthesis. Free Radic Biol Med. 1999;(9-10): 922-935.

11. Witztum JL, Steinberg D. Role of oxidized low density lipoprotein in atherogenesis. J Clin Invest. 1991;88(6):1785-1792.

12. Rosenblat M, Volkova N, Coleman R, Aviram M. Anti-oxidant and antiatherogenic properties of liposomal glutathione: studies in vitro, and in the atherosclerotic apolipoprotein E-deficient mice. Atherosclerosis. 2007;195(2):e61-e68.

13. Fimognari FL, Scarlata S, Conte ME, Incalzi RA. Mec hanisms of atherothrombosis in chronic obstructive pulmonary disease. Int J Chron Obstruct Pulmon Dis. 2008;3(1):89-96.

14. Colles SM, Maxson JM, Carlson SG, Chisolm GM. Oxidized LDLinduced injury and apoptosis in atherosclerosis. Potential roles for oxysterols. Trends Cardiovasc Med. 2001;11(3-4):131-138.

15. Berliner JA, Territo MC, Sevanian A, Ramin S, Kim JA, Bamshad B, et al. Minimally modified low density lipoprotein stimulates monocyte endothelial interactions. J Clin Invest. 1990;85(4):1260-1266.

16. Thomas ED, Ramberg RE, Sale GE, Sparkes RS, Golde DW. Direct evidence for a bone marrow origin of the alveolar macrophage in man Science. 1976;192(4243):1016-1018.

17. Cline MJ, Lehrer RI, Territo MC, Golde DW. UCLA Conference Monocytes and macrophages: functions and diseases. Ann Intern Med. 1978;88(1):78-88.
18. Ping XD, Harris FL, Brown LA, Gauthier TW. In vivo dysfunction of the term alveolar macrophage after in utero ethanol exposure. Alcohol Clin Exp Res. 2007;31(2):308-316.

19. Deng T, Zhang L, Ge Y, Lu M, Zheng X. Redistribution of intracellular calcium and its effect on apoptosis in macrophages: Induction by oxidized LDL. Biomed Pharmacother. 2008;63(4):267-274.

20. Qiao M, Kisgati M, Cholewa JM, Zhu W, Smart EJ, Sulistio MS, et al. Increased expression of glutathione reductase in macrophages decreases atherosclerotic lesion formation in low-density lipoprotein receptor-deficient mice. Arterioscler Thromb Vasc Biol. 2007;27(6): 1375-1382.

21. Hamilos DL, Zelarney P, Mascali JJ. Lymphocyte proliferation in glutathione-depleted lymphocytes: direct relationship between glutathione availability and the proliferative response. Immunopharmacology. 1989;18(3):223-235.

22. Messina JP, Lawrence DA. Cell cycle progression of glutathionedepleted human peripheral blood mononuclear cells is inhibited at S phase. J Immunol. 1989;143(6):1974-1981.

23. Cantin AM, North SL, Hubbard RC, Crystal RG. Normal alveolar epithelial lining fluid contains high levels of glutathione. JAppl Physiol. 1987;63(1):152-157.

24. Brown LAS, Ping X-D, Harris FL, Gauthier TW. Glutathione availability modulates alveolar macrophage function in the chronic ethanol-fed rat. Am J Physiol Lung Cell Mol Physiol. 2007;292(4): L824-L832.

25. Stocker R, Keaney JF Jr. Role of oxidative modifications in atherosclerosis. Physiol Rev. 2004;84(4):1381-1478.

26. Hansel TT, Barnes PJ. Recent Advances in the Pathophysiology of COPD. Basel Boston Birkhäuser Verlag. 2004.

27. Rahman I, MacNee W. Oxidative stress and regulation of glutathione in lung inflammation. Eur Respir J. 2000;16(3):534-554.

28. Barnes PJ. Oxidative stress in COPD. in Hansel TT, Barnes PJ, eds. Recent Advances in the Pathophysiology of COPD. Basel; Boston: Birkhäuser Verlag. 2004.

29. Loeb GA, Skelton DC, Coates TD, Forman HJ. Role of seleniumdependent glutathione peroxidase in antioxidant defenses in rat alveolar macrophages. Exp Lung Res. 1988;14 Suppl:921-936.

30. Pietarinen P, Raivio K, Devlin RB, Crapo JD, Chang LY, Kinnula VL. Catalase and glutathione reductase protection of human alveolar macrophages during oxidant exposure in vitro. Am J Respir Cell Mol Biol. 1995;13(4):434-441.

31. Zhang W, Salomon RG. Oxidized phospholipids, isolevuglandins, and atherosclerosis. Mol Nutr Food Res. 2005;49(11):1050-1062.

32. Wintergerst ES, Jelk J, Rahner C, Asmis R. Apoptosis induced by oxidized low density lipoprotein in human monocyte-derived macrophages involves CD36 and activation of caspase-3. Eur J Biochem. 2000;267(19):6050-6059.

33. Wang Y, Qiao M, Mieyal JJ, Asmis LM, Asmis R. Molecular mechanism of glutathione-mediated protection from oxidized lowdensity lipoprotein-induced cell injury in human macrophages: role of glutathione reductase and glutaredoxin. Free Radic Biol Med. 2006;41(5):775-785.

34. Pedersen NC, Ho E, Brown ML, Yamamoto JK. Isolation of a T-lymphotropic virus from domestic cats with an immunodeficiency-like syndrome. Science. 1987;235:790-793.

35. Venketaraman V, Rodgers $\mathrm{T}$, Linnares $\mathrm{R}$, et al. Tuberculosis immunity in healthy and HIV-infected subjects. AIDS Res Ther. 2006;3(1):5.

36. Buhl R, Jaffe HA, Holroyd KJ, et al. Systemic glutathione deficiency in symptom-free HIV-seropositive individuals. Lancet. 1989;2: 1294-1298.

37. de Quay B, Malinverni R, Lauterburg BH. Glutathione depletion in HIV-infected patients: role of cysteine deficiency and effect of oral N-acetylcysteine. AIDS. 1992;6:815-819.

38. Eck HP, Gmunder H, Hartmann M, Petzoldt D, Daniel V, Droge W. Low concentrations of acid-soluble thiol (cysteine) in the blood plasma of HIV-1-infected patients. Biol Chem Hoppe Seyler. 1989;370: 101-108. 
39. Helbling B, von Overbeck J, Lauterburg BH. Decreased release of glutathione into the systemic circulation of patients with HIV infection. Eur J Clin Invest. 1996;26:38-44.

40. Pantaleo G, Graziosi C, Fauci AS. New concepts in the immunopathogenesis of human immuno deficiency infection. $N$ Engl J Med. 1993; 328:327-335.

41. Levy JA. HIV pathogenesis and long term survival. AIDS. 1993;7: 1401-1410

42. Droge W, Holm E. Role of cysteine and glutathione in HIV infection and other diseases associated with muscle wasting and immunological dysfunction. FASEB J. 1997;11:1077-1089.

43. Herzenberg LA, de Rosa SC, Dubs JG, et al. Glutathione deficiency is associated with impaired survival in HIV disease. Proc Natl Acad Sci US A. 1997;94:1967-1972.

44. Staal FJ. Glutathione and HIV infection: reduced reduced or increased oxidized? Eur J Clin Invest. 1998;2:194-196.

45. Tsao TC, Hong J, Li LF, Hsieh MJ, Liao SK, Chang KS. Imbalances between tumor necrosis factor-alpha and its soluble receptor forms, and interleukin-1beta and interleukin-1 receptor antagonist in BAL fluid of cavitary pulmonary tuberculosis. Chest. 2000;117:103-109.

46. Hag AM, Kristoffersen US, Pedersen SF, et al. Regional gene expression of LOX-1, VCAM-1, and ICAM-1 in aorta of HIV-1 transgenic rats. PloS One. 2009; 4:e8170.

47. Dayaram YK, Talaue MT, Connell ND, Venketaraman V. Characterization of a glutathione metabolic mutant of Mycobacterium tuberculosis and its resistance to glutathione and nitrosoglutathione. J Bacteriol. 2006;188(4):1364-1372.

48. Brill KJ, Li Q, Larkin R, et al. Human natural killer cells mediate killing of intracellular Mycobacterium tuberculosis H37Rv via granuleindependent mechanisms. Infect Immun. 2001;69(3):1755-1765.

49. Chan ED, Chan J, Schluger NW. What is the role of nitric oxide in murine and human host defense against tuberculosis? Current knowledge. Am J Respir Cell Mol Biol. 2001;25(5):606-612.

50. Flynn JL, Chan J, Triebold KJ, Dalton DK, Stewart TA, Bloom BR. An essential role for interferon- $\alpha$ in resistance to Mycobacterium tuberculosis infection. J Exp Med. 1993;178:2249-2252.

51. Venketaraman V, Dayaram YK, Amin AG, et al. Role of glutathione in macrophage control of mycobacteria. Infect Immun. 2003;71(4): 1864-1871

52. Venketaraman V, Dayaram YK, Talaue MT, Connell ND. Glutathione and nitrosoglutathione in macrophage defense against. M. tuberculosis. Infect Immun. 2005;73(3):1886-1894.

53. Millman AC, Salman M, Dayaram YK, Connell ND, Venketaraman V. Natural killer cells, glutathione, cytokines and innate immunity against Mycobacterium tuberculosis. J Interferon Cytokine Res. 2008;28: 1-13.
54. Venketaraman V, Millman AC, Salman M, et al. Glutathione levels and immune responses in tuberculosis patients. Microb Pathog. 2008;44:255-261.

55. Gutteridge JM. Lipid peroxidation and antioxidants as biomarkers of tissue damage. Clin Chem. 1995;41(12 Pt 2):1819-1828.

56. Schulze-Osthoff K, Los M, Baeuerle PA. Redox signalling by transcription factors NF-kappa B and AP-1 in lymphocytes. Biochem Pharmacol. 1995;50:735-741.

57. Dubey SS, Sinha KK, Gupta JP. Vitamin C status, glutathione and histamine in gastric carcinoma, tuberculous enteritis and nonspecific ulcerative colitis. Indian J Physiol Pharmacol. 1985;29(2): 111-114

58. Kwiatkowska S, Piasecka G, Zieba M, Piotrowski W, Nowak D. Increased serum concentrations of conjugated diens and malondialdehyde in patients with pulmonary tuberculosis. Respir Med. 1999;93(4): $272-276$.

59. Jack CI, Jackson MJ, Hind CR. Circulating markers of free radical activity in patients with pulmonary tuberculosis. Tuber Lung Dis. 1994; 75(2):132-137.

60. Tsao TC, Hong J, Li LF, Hsieh MJ, Liao SK, Chang KS. Imbalances between tumor necrosis factor-alpha and its soluble receptor forms, and interleukin-1beta and interleukin-1 receptor antagonist in BAL fluid of cavitary pulmonary tuberculosis. Chest. 2000;117(1):103-109.

61. Makonkawkeyoon S, Limson Pobre RN, Moreira AL, Schauf V, Kaplan G. Thalidomide inhibits the replication of human immunodeficiency virus type 1. Proc Natl Acad Sci U S A. 1993;90:5974-5978.

62. Wallis RS, Nsubuga P, Okwera A, et al. Pentoxifylline in human immunodeficiency virus-seropositive tuberculosis: a randomized, controlled trial. J Infect Dis. 1996;174(4):727-733.

63. Mayanja-Kizza H, Jones-Lopez E, Okwera A, et al. Uganda-Case Western Research Collaboration. Immunoadjuvant prednisolone therapy for HIV-associated tuberculosis: a phase 2 clinical trial in Uganda. J Infect Dis. 2005;191(6):856-865.

64. Wallis RS, Kalayjian R, Jacobson JM, et al. A study of the immunology, virology, and safety of prednisone in HIV-1-infected subjects with CD4 cell counts of 200 to $700 \mathrm{~mm}(-3)$. J Acquir Immune Defic Syndr. 2003;32(3):281-286.

65. van Crevel R, Ottenhoff $\mathrm{TH}$, van der Meer JW. Innate immunity to Mycobacterium tuberculosis. Clin Microbiol Rev. 2002;15(2): 294-309.

66. Christman MA 2nd, Goetz DJ, Dickerson E, et al. Wnt5a is expressed in murine and human atherosclerotic lesions. $J$ Physiol Heart Circ Physiol. 2008;294(6):H2864-H2870.
HIV/AIDS - Research and Palliative Care

\section{Publish your work in this journal}

HIV/AIDS - Research and Palliative Care is an international, peerreviewed open-access journal focusing on advances in research in HIV, its clinical progression and management options including antiviral treatment, palliative care and public healthcare policies to control viral spread. The journal welcomes original research, basic science,

\section{Dovepress}

clinical \& epidemiological studies, reviews \& evaluations, expert opinion \& commentary, case reports \& extended reports. The manuscript management system is completely online and includes a very quick and fair peer-review system. Visit http://www.dovepress.com/ testimonials.php to read real quotes from published authors. 